\title{
ON THE CONVERGENCE RATE OF A MODIFIED FOURIER SERIES
}

\author{
SHEEHAN OLVER
}

\begin{abstract}
The rate of convergence for an orthogonal series that is a minor modification of the Fourier series is proved. This series converges pointwise at a faster rate than the Fourier series for nonperiodic functions. We present the error as an asymptotic expansion, where the lowest term in this expansion is of asymptotic order two. Subtracting out the terms from this expansion allows us to increase the order of convergence, though the terms of this expansion depend on derivatives. Alternatively, we can employ extrapolation methods which achieve higher convergence rates using only the coefficients of the series. We also present a method for the efficient computation of the coefficients in the series.
\end{abstract}

\section{INTRODUCTION}

The standard Fourier series serves as the bedrock of much of modern applied mathematics. Its utility is ubiquitous: from signal analysis to solving differential equations. The discrete counterpart, in particular the Fast Fourier Transform (FFT), has revolutionized numerical analysis. The Fourier series over the interval $[-1,1]$, written here in terms of the real cosine and sine functions, is:

$$
f(x) \sim \frac{c_{0}}{2}+\sum_{k=1}^{\infty}\left[c_{k} \cos \pi k x+d_{k} \sin \pi k x\right]
$$

for

$$
c_{k}=\langle f, \cos \pi k x\rangle=\int_{-1}^{1} f(x) \cos \pi k x \mathrm{~d} x
$$

and

$$
d_{k}=\langle f, \sin \pi k x\rangle=\int_{-1}^{1} f(x) \sin \pi k x \mathrm{~d} x .
$$

Part of the Fourier series' importance rests on the fact that, when $f$ is analytic and periodic, the series converges exponentially fast. Thus only a very small number of terms need to be computed in order to obtain an accurate approximation. But as soon as $f$ loses its periodicity, the convergence rate of the $n$-term partial sum drops drastically to $\mathcal{O}\left(n^{-1}\right)$. Though the order of convergence can be increased by subtracting out a polynomial, this suffers from the fact that the expansion no longer has an orthogonal basis.

Received by the editor April 22, 2008.

2000 Mathematics Subject Classification. Primary 42A20.

Key words and phrases. Orthogonal series, function approximation, oscillatory quadrature.

(C)2009 American Mathematical Society Reverts to public domain 28 years from publication 
In [8, it was noted that with a slight alteration to the Fourier series we obtain a series that converges for nonperiodic differentiable functions, and whose coefficients decay like $\mathcal{O}\left(k^{-2}\right)$. In Section 2, we give a brief overview of what is known about this modified Fourier series. It was demonstrated numerically that the $n$-term partial sums of the series appear to approximate the original function with an

error of order $\mathcal{O}\left(n^{-2}\right)$. In Section 3, we will prove this result, assuming that the function is sufficiently differentiable. The error of the partial sums is written as an asymptotic expansion, which means that subtracting out a simple correction term allows us to increase the order of convergence. Asymptotic expansions lend themselves to extrapolation methods for convergence acceleration. This is explored in Section 4. where a Richardson-like extrapolation method is developed, as well as an extrapolation method based on interpolation. Combining the two methods results in a convergence acceleration scheme that depends only on the coefficients of the series.

The usefulness of this series relies on the computation of the coefficients, which are highly oscillatory integrals. Though traditionally considered difficult to compute, modern advances have shattered this view. In fact, methods exist which actually increase with accuracy as the frequency of the oscillations increases. This is similar in behaviour to an asymptotic expansion, though significantly more accurate. In Section 5 we give a brief overview of one of these methods and show how it can be utilized for the modified Fourier series constructed in Section 2.

\section{MOdIFIED Fourier SERIES}

This section is based on material found in 8 . Consider for a moment the standard Fourier series. When $f$ is sufficiently differentiable, we can determine the order of decay of the coefficients with a straightforward application of integration by parts:

$$
\begin{aligned}
c_{k} & =-\frac{1}{\pi k} \int_{-1}^{1} f^{\prime}(x) \sin \pi k x \mathrm{~d} x \\
& =\frac{(-1)^{k}}{(\pi k)^{2}}\left[f^{\prime}(1)-f^{\prime}(-1)\right]-\frac{1}{(\pi k)^{2}} \int_{-1}^{1} f^{\prime \prime}(x) \cos \pi k x \mathrm{~d} x=\mathcal{O}\left(k^{-2}\right), \\
d_{k} & =\frac{(-1)^{k+1}}{\pi k}[f(1)-f(-1)]+\frac{1}{\pi k} \int_{-1}^{1} f^{\prime}(x) \cos \pi k x \mathrm{~d} x=\mathcal{O}\left(k^{-1}\right) .
\end{aligned}
$$

Interestingly, only the sine terms decay like $\mathcal{O}\left(k^{-1}\right)$; the cosine terms decay at the faster rate of $\mathcal{O}\left(k^{-2}\right)$. Indeed, if $f$ is even, then all the sine terms are zero and the rate of convergence increases. In the construction of the modified Fourier series, we replace the sine terms with an alternate basis such that the orthogonality property is still maintained, whilst the order of decay of the coefficients is increased to $\mathcal{O}\left(k^{-2}\right)$.

Consider the expansion in the series

$$
f(x) \sim \frac{c_{0}}{2}+\sum_{k=1}^{\infty}\left[c_{k} \cos \pi k x+s_{k} \sin \pi\left(k-\frac{1}{2}\right) x\right],
$$


where

$$
\begin{aligned}
& c_{k}=\langle f, \cos \pi k x\rangle=\int_{-1}^{1} f(x) \cos \pi k x \mathrm{~d} x, \\
& s_{k}=\left\langle f, \sin \pi\left(k-\frac{1}{2}\right) x\right\rangle=\int_{-1}^{1} f(x) \sin \pi\left(k-\frac{1}{2}\right) x \mathrm{~d} x .
\end{aligned}
$$

We will denote the $n$-term partial sum of this series by $f_{n}$ :

$$
f_{n}(x)=\frac{c_{0}}{2}+\sum_{k=1}^{n}\left[c_{k} \cos \pi k x+s_{k} \sin \pi\left(k-\frac{1}{2}\right) x\right] .
$$

Like the standard Fourier series, it can be shown that the basis functions of this series are orthogonal. This follows since they are the eigenfunctions of the differential equation

$$
u^{\prime \prime}+\alpha^{2} u=0, \quad 0=u^{\prime}(1)=u^{\prime}(-1) .
$$

Unlike the Fourier series, the new sine terms are not periodic with respect to the interval $[-1,1]$; hence we can consider this series for approximating nonperiodic functions.

If $f \in C^{2 s}[-1,1]$ and $f^{(2 s)}$ has bounded variation, then Theorem 13.2 in [12] tells us that

$$
\int_{-1}^{1} f^{(2 s)}(x) e^{i \pi k x} \mathrm{~d} x=\mathcal{O}\left(k^{-1}\right) .
$$

In this case the asymptotic expansion for the coefficients can be found by repeatedly integrating by parts in the same manner as in (2.1), giving us, for $k \neq 0$ :

$$
\begin{aligned}
& c_{k}=\sum_{j=1}^{s} \frac{(-1)^{k+j+1}}{(\pi k)^{2 j}}\left[f^{(2 j-1)}(1)-f^{(2 j-1)}(-1)\right]+\mathcal{O}\left(k^{-2 s-1}\right), \\
& s_{k}=\sum_{j=1}^{s} \frac{(-1)^{k+j}}{(\pi(k-1 / 2))^{2 j}}\left[f^{(2 j-1)}(1)+f^{(2 j-1)}(-1)\right]+\mathcal{O}\left(k^{-2 s-1}\right) .
\end{aligned}
$$

The cosine terms $c_{k}$ are exactly the same as in the standard Fourier series, hence still decay like $\mathcal{O}\left(k^{-2}\right)$. The new sine terms $s_{k}$, unlike the standard Fourier series sine terms $d_{k}$, also decay like $\mathcal{O}\left(k^{-2}\right)$. Since the coefficients go to zero at a faster rate, it stands to reason that the series itself will converge to the actual function more rapidly than the standard Fourier series. This will be proved to be the case in Section 3 .

In order to prove the rate of convergence, we first need to know that the series does indeed converge pointwise. This is not trivial, but thankfully was proved in [8]:

Theorem 2.1 ([8]). The modified Fourier series forms an orthonormal basis of $L_{2}[-1,1]$.

Theorem $2.2([8])$. Suppose that $f$ is a Riemann integrable function in $[-1,1]$ and that

$$
c_{k}, s_{k}=\mathcal{O}\left(n^{-1}\right), \quad n \rightarrow \infty .
$$

If $f$ is Lipshitz at $x \in(-1,1)$, then

$$
f_{n}(x) \rightarrow f(x) .
$$



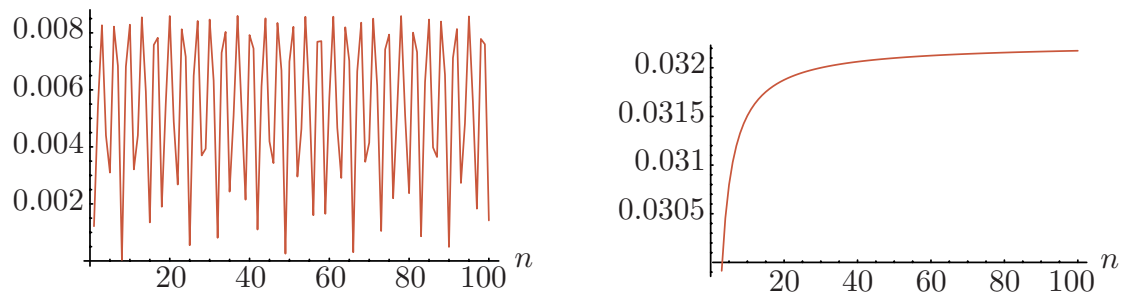

Figure 1. For $f(x)=\operatorname{Ai}(x)$, the error $\left|f(-1 / \sqrt{2})-f_{n}(-1 / \sqrt{2})\right|$ scaled by $n^{2}$ (left graph) and the error $\left|f(1)-f_{n}(1)\right|$ scaled by $n$ (right graph).

The convergence at the endpoints in [8] was proved only for the case when $f$ is analytic. Thus we present an alternate proof that enlarges the class of functions such that pointwise convergence is known:

Theorem 2.3. Suppose that $f \in C^{2}[-1,1]$ and $f^{\prime \prime}$ has bounded variation. Then

$$
f_{n}( \pm 1) \rightarrow f( \pm 1) .
$$

Proof. We know that the coefficients of the series decay like $\mathcal{O}\left(k^{-2}\right)$; thus

$$
\sum_{k=1}^{\infty}\left(\left|c_{k}\right|+\left|s_{k}\right|\right)<\infty
$$

and $f_{n}$ converges uniformly to a continuous function. Two continuous functions that differ at a single point must differ in a neighbourhood of that point. The theorem thus follows from Theorem 2.2. since $f$ is Lipshitz everywhere.

We can demonstrate the accuracy of this expansion numerically. Consider the Airy function $\operatorname{Ai}(x)$ [1. Since the turning point of the Airy function is within $[-1,1]$, it behaves very differently on the negative segment of the interval than it does on the positive segment. Despite this difficulty, we can still approximate the function efficiently. This can be seen in Figure 1, which demonstrates the error of the $n$-term partial sum $f_{n}$ at the points $x=-1 / \sqrt{2}$ and $x=1$. We intentionally chose an irrational number to demonstrate generality, as the proof of the convergence rate relies on a special function that has a simpler form at rational points. As can be seen, the error does appear to decay like $\mathcal{O}\left(n^{-2}\right)$ at the chosen interior point, while it decays at the reduced rate of $\mathcal{O}\left(n^{-1}\right)$ at the right endpoint.

As another example, consider the function $f(x)=2 /\left(7+20 x+20 x^{2}\right)$. This function suffers from Runge's phenomenon [14, where $f$ has poles in the complex plane near the real axis, which makes the function notoriously difficult to approximate. Figure 2 shows that the modified Fourier series is still very accurate and compares its accuracy to that of the standard Fourier series. We see the error of the partial sums over the entire interval, for four choices of $n$. As can be seen, the modified Fourier series approximates inside the interior of the interval significantly better than it does at the endpoints. With as little as 60 terms we obtain an error of about $2 \times 10^{-5}$ around zero, compared to an error of about $10^{-3}$ for the standard Fourier series. Furthermore, unlike the standard Fourier series, there is no Gibbs' phenomenon at the boundary points. 

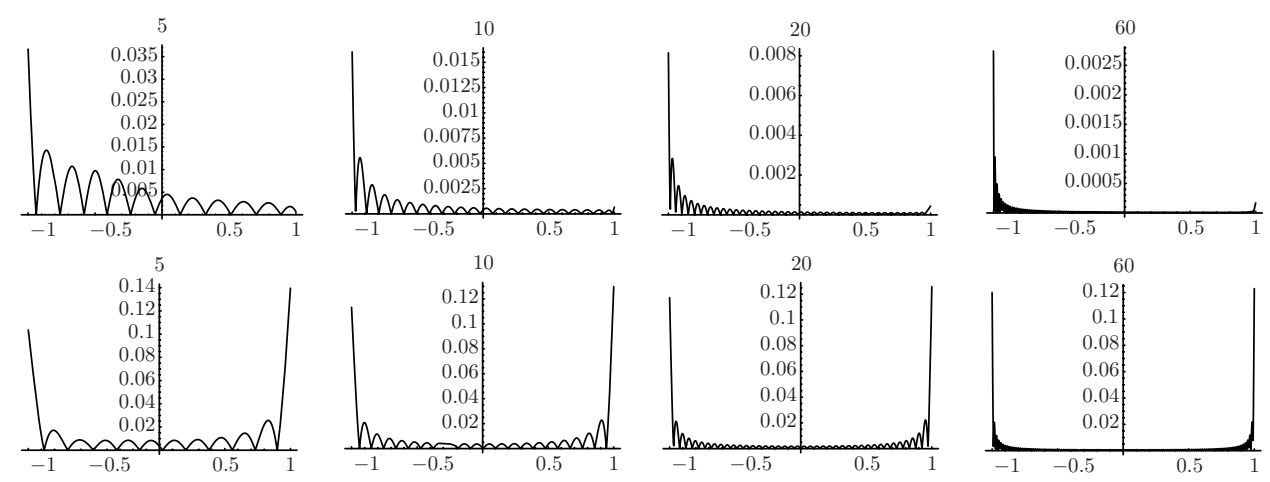

FiguRE 2. For $f(x)=2 /\left(7+20 x+20 x^{2}\right)$, the error $\left|f(x)-f_{n}(x)\right|$ (top graphs), versus the error of the $n$-term partial sums of the Fourier series (bottom graphs), for $n=$ $5,10,20,60$.

\section{Convergence Rate}

Though Iserles and Nørsett 8 proved the convergence of the modified Fourier series and demonstrated numerically that it converges at a rate of $\mathcal{O}\left(n^{-2}\right)$, they failed to prove this convergence rate, even for the case where $f$ is analytic. In this section, we present a proof for the case when $f \in C^{2}[-1,1]$ and $f^{\prime \prime}$ has bounded variation. Furthermore, we prove this result in such a way that, assuming sufficient differentiability of $f$, we know explicitly the error term that decays like $\mathcal{O}\left(n^{-2}\right)$, meaning we can actually increase the order of approximation by subtracting out this term. In fact, if $f$ is smooth, we can expand the error $f(x)-f_{n}(x)$ in terms of an asymptotic expansion for $n \rightarrow \infty$, meaning that by subtracting out a correction term we can increase the order of approximation to be arbitrarily high. This expansion is even valid at the endpoints of the interval.

The proof involves writing the error itself in terms of its modified Fourier series, replacing the coefficients of this series by the first $s$-terms of their asymptotic expansions, and using a known special function, specifically the Lerch transcendent function $\Phi$ [15], to rewrite the resulting infinite sum. The Lerch transcendent function is defined as

$$
\Phi(z, s, m)=\sum_{k=0}^{\infty} \frac{z^{k}}{(k+m)^{s}} .
$$

We begin by defining a couple of key properties of the asymptotics of $\Phi$ :

Lemma 3.1. Suppose that $s \geq 2$ and define

$$
\sigma_{x, s}(t)=\frac{t^{s-1}}{1+e^{i \pi x-t}}
$$

If $-1<x<1$, then

$$
\Phi\left(-e^{i \pi x}, s, m\right) \sim \frac{1}{\Gamma(s)} \sum_{k=s}^{\infty} \frac{\sigma_{x, s}^{(k-1)}(0)}{m^{k}}, \quad m \rightarrow \infty .
$$


If $x= \pm 1$, then

$$
\Phi(1, s, m) \sim \frac{1}{\Gamma(s)} \sum_{k=s-1}^{\infty} \lim _{t \rightarrow 0} \frac{\sigma_{ \pm 1, s}^{(k-1)}(t)}{m^{k}}, \quad m \rightarrow \infty .
$$

Proof. We first consider the case where $-1<x<1$. It is clear that $0=\sigma_{x, s}(0)=$ $\sigma_{x, s}^{\prime}(0)=\cdots=\sigma_{x, s}^{(s-2)}(0)$. We know from [15] that, whenever $-e^{i \pi x} \neq 1$, the Lerch transcendent function has the integral representation

$$
\Phi(z, s, m)=\frac{1}{\Gamma(s)} \int_{0}^{\infty} \frac{t^{s-1}}{1-z e^{-t}} e^{-m t} \mathrm{~d} t .
$$

Furthermore,

$$
\int_{0}^{\infty} \sigma_{x, s}^{(k)}(t) e^{-m t} \mathrm{~d} t=\frac{1}{m} \sigma_{x, s}^{(k)}(0)+\frac{1}{m} \int_{0}^{\infty} \sigma_{x, s}^{(k+1)}(t) e^{-m t} \mathrm{~d} t .
$$

The first part of the theorem thus follows from induction, since

$$
\Phi\left(-e^{i \pi x}, s, m\right)=\int_{0}^{\infty} \sigma_{x, s}(t) e^{-m t} \mathrm{~d} t .
$$

For the case when $x= \pm 1$, hence $-e^{i \pi x}=1$, the Lerch transcendent reduces to the Hurwitz zeta function: $\Phi(1, s, m)=\zeta(s, m)[1$. This has the same integral representation; however, a singularity is now introduced at zero:

$$
\zeta(s, m)=\frac{1}{\Gamma(s)} \int_{0}^{\infty} \frac{t^{s-1}}{1-e^{-t}} e^{-m t} \mathrm{~d} t=\frac{1}{\Gamma(s)} \int_{0}^{\infty} \sigma_{ \pm 1, s}(t) e^{-m t} \mathrm{~d} t .
$$

With a bit of care, however, we can integrate by parts in the same manner. For this choice of $x$ we obtain

$$
\sigma_{ \pm 1, s}(t)=\frac{t^{s-1}}{1-e^{-t}}
$$

hence, due to L'Hôpital's rule, only the first $s-3$ derivatives of $\sigma_{ \pm 1, s}$ vanish at zero. But $\sigma_{ \pm 1, s}$ is still $C^{\infty}$ at zero, since

$$
\sigma_{ \pm 1, s}(t)=\frac{t^{s-1}}{1-e^{-t}}=\frac{t^{s-1}}{t\left(1+\mathcal{O}\left(t^{2}\right)\right)}=\frac{t^{s-2}}{1+\mathcal{O}\left(t^{2}\right)} .
$$

Thus integration by parts gives us its asymptotic expansion.

With the Lerch transcendent function in hand, we can successfully find the asymptotic expansion for the error of the modified Fourier series:

Theorem 3.2. Suppose that $f \in C^{2 s}[-1,1]$ and $f^{(2 s)}$ has bounded variation. Then

$$
\begin{aligned}
f(x)-f_{n}(x)=\frac{1}{2} \sum_{j=1}^{s} & \frac{(-1)^{j}}{\pi^{2 j}}\left[\left(f^{(2 j-1)}(1)-f^{(2 j-1)}(-1)\right) \tilde{\Phi}_{1}(x, j, n)\right. \\
& \left.+i\left(f^{(2 j-1)}(1)+f^{(2 j-1)}(-1)\right) \tilde{\Phi}_{2}(x, j, n)\right]+\mathcal{O}\left(n^{-2 s}\right),
\end{aligned}
$$

for

$$
\begin{aligned}
\tilde{\Phi}_{1}(x, j, n)=(-1)^{n}\left[e^{i \pi(n+1) x} \Phi\left(-e^{i \pi x}, 2 j, n+1\right)\right. \\
\\
\left.\quad+e^{-i \pi(n+1) x} \Phi\left(-e^{-i \pi x}, 2 j, n+1\right)\right]
\end{aligned}
$$


and

$$
\begin{aligned}
& \tilde{\Phi}_{2}(x, j, n)=(-1)^{n}\left[e^{i \pi\left(n+\frac{1}{2}\right) x} \Phi\left(-e^{i \pi x}, 2 j, n+\frac{1}{2}\right)\right. \\
&\left.-e^{-i \pi\left(n+\frac{1}{2}\right) x} \Phi\left(-e^{-i \pi x}, 2 j, n+\frac{1}{2}\right)\right] .
\end{aligned}
$$

Proof. Note that

$$
f(x)-f_{n}(x)=\sum_{k=n+1}^{\infty}\left[c_{k} \cos \pi k x+s_{k} \sin \pi\left(k-\frac{1}{2}\right) x\right] .
$$

This follows from the pointwise convergence of the modified Fourier series. We define the following two constants for brevity:

$A_{j}^{C}=(-1)^{j} \frac{f^{(2 j-1)}(1)-f^{(2 j-1)}(-1)}{2 \pi^{2 j}}, \quad A_{j}^{S}=(-1)^{j+1} \frac{f^{(2 j-1)}(1)+f^{(2 j-1)}(-1)}{2 i \pi^{2 j}}$.

Substituting in the asymptotic expansion for the coefficients (2.2), the error of the partial sum is equal to

$$
\begin{aligned}
f(x)-f_{n}(x)=-\sum_{j=1}^{s}\left[A_{j}^{C} \sum_{k=n+1}^{\infty} \frac{(-1)^{k}}{k^{2 j}}\left(e^{i \pi k x}+e^{-i \pi k x}\right)\right. & \\
& \left.+A_{j}^{S} \sum_{k=n+1}^{\infty} \frac{(-1)^{k}}{\left(k-\frac{1}{2}\right)^{2 j}}\left(e^{i \pi\left(k-\frac{1}{2}\right) x}-e^{-i \pi\left(k-\frac{1}{2}\right) x}\right)\right] \\
& +\sum_{k=n+1}^{\infty} \mathcal{O}\left(k^{-2 s-1}\right) \\
=\sum_{j=1}^{s}(-1)^{n}\left[A _ { j } ^ { C } \left\{e^{i \pi(n+1) x} \sum_{k=0}^{\infty} \frac{(-1)^{k}}{(k+n+1)^{2 j}} e^{i \pi k x}\right.\right. & \left.+e^{-i \pi(n+1) x} \sum_{k=0}^{\infty} \frac{(-1)^{k}}{(k+n+1)^{2 j}} e^{-i \pi k x}\right\} \\
& +A_{j}^{S}\left\{e^{i \pi\left(n+\frac{1}{2}\right) x} \sum_{k=0}^{\infty} \frac{(-1)^{k}}{\left(k+n+\frac{1}{2}\right)^{2 j}} e^{i \pi k x}\right. \\
+ & \mathcal{O}\left(n^{-2 s}\right) .
\end{aligned}
$$

By replacing the infinite sums with their Lerch transcendent function equivalent, we obtain

$$
f(x)-f_{n}(x)=\sum_{j=1}^{s}\left[A_{j}^{C} \tilde{\Phi}_{1}(x, j, n)+A_{j}^{S} \tilde{\Phi}_{2}(x, j, n)\right]+\mathcal{O}\left(n^{-2 s}\right) .
$$

Remark. If $f \in C^{2 s+1}[-1,1]$ in the preceding theorem, then the order of the error term increases to $\mathcal{O}\left(n^{-2 s-1}\right)$. This follows since we can integrate by parts once more in the asymptotic expansion of the coefficients, meaning the $s$-term expansion now has an order of error $\mathcal{O}\left(n^{-2 s-2}\right)$. We could have also written the cosine and sine 

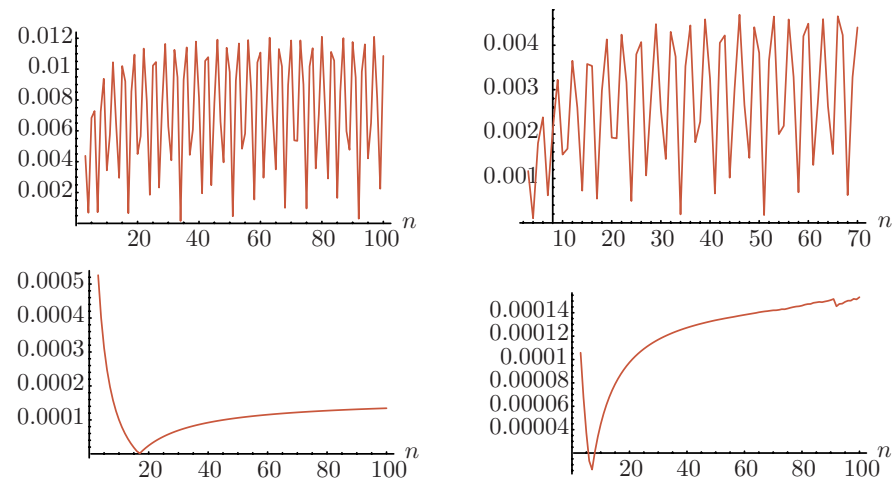

FiguRE 3. For $f(x)=\operatorname{Ai}(x)$, the error approximating $f(-1 / \sqrt{2})$ by $f_{n}(-1 / \sqrt{2})+E_{1, n}(-1 / \sqrt{2})$ scaled by $n^{4}$ (top left), $f(-1 / \sqrt{2})$ by $f_{n}(-1 / \sqrt{2})+E_{2, n}(-1 / \sqrt{2})$ scaled by $n^{6}$ (top right), $f(1)$ by $f_{n}(1)+E_{1, n}(1)$ scaled by $n^{3}$ (bottom left) and $f(1)$ by $f_{n}(1)+$ $E_{2, n}(1)$ scaled by $n^{5}$ (bottom right).

functions as the real and imaginary parts of $e^{i x}$, which would have resulted in two fewer evaluations of the Lerch transcendent function.

Substituting the Lerch transcendent functions in this theorem with the asymptotic expansion found in Lemma 3.1 results in an asymptotic expansion for the error in terms of elementary functions. Unfortunately this expansion explodes near the endpoints, even though another expansion in terms of elementary functions is known at the endpoints. Thus, since the Lerch transcendent functions can be computed efficiently [2, it is preferred to leave the Lerch transcendent functions in their unexpanded form.

As a result of this theorem, we obtain a sufficient condition for obtaining a rate of convergence of order two:

Corollary 3.3. Suppose that $f \in C^{2}[-1,1]$ and $f^{\prime \prime}$ has bounded variation. If $-1<x<1$, then

Otherwise,

$$
f(x)-f_{n}(x)=\mathcal{O}\left(n^{-2}\right) .
$$

$$
f( \pm 1)-f_{n}( \pm 1)=\mathcal{O}\left(n^{-1}\right) .
$$

Proof. This corollary follows immediately by combining Theorem 3.2 with the asymptotic behaviour of $\Phi$ found in Lemma 3.1 .

Define the $s$-term expansion of the error as

$$
\begin{aligned}
E_{s, n}(x)=\frac{1}{2} \sum_{j=1}^{s} \frac{(-1)^{j}}{\pi^{2 j}}[ & \left(f^{(2 j-1)}(1)-f^{(2 j-1)}(-1)\right) \tilde{\Phi}_{1}(x, j, n) \\
& \left.+i\left(f^{(2 j-1)}(1)+f^{(2 j-1)}(-1)\right) \tilde{\Phi}_{2}(x, j, n)\right] .
\end{aligned}
$$

As an example, consider again the function $\mathrm{Ai}(x)$. In Figure 3, we demonstrate that subtracting out the error term $E_{s, n}(x)$ does indeed increase the order of approximation. In the first two graphs of this figure, we see that this is true for the 

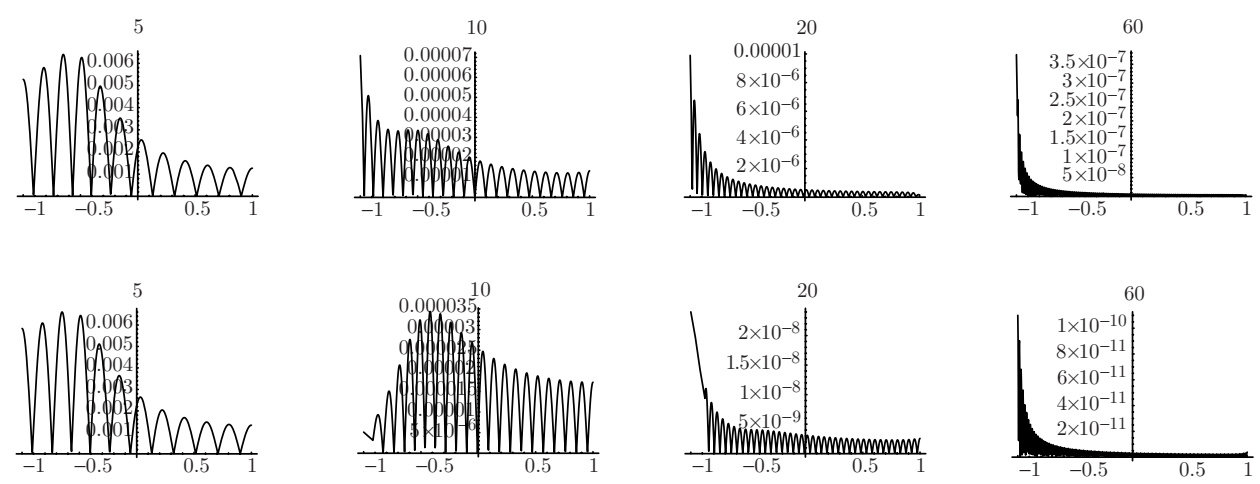

Figure 4. For $f(x)=2 /\left(7+20 x+20 x^{2}\right)$, the error approximating $f(x)$ by $f_{n}(x)+E_{1, n}(x)$ (top graphs) and by $f_{n}(x)+E_{2, n}(x)$ (bottom graphs), for $n=5,10,20,60$.

interior point $x=-1 / \sqrt{2}$, where subtracting out $E_{1, n}(x)$ increases the order to $n^{4}$ and subtracting out $E_{2, n}(x)$ increases the order further to $n^{6}$. In the second graph we end the data at $n=60$, as we have almost reached machine precision. Since $\mathrm{Ai}(x)$ is analytic, we could continue this process to obtain arbitrarily high orders. The last two graphs show that we can employ the same technique at the boundary point $x=1$, where the order is first increased to $n^{3}$, then to $n^{5}$.

We can see how subtracting out the first term in the error expansion affects the approximation over the entire interval. In Figure 4, we return to the example first presented in Figure 2, where $f(x)=2 /\left(7+20 x+20 x^{2}\right)$. As can be seen, we obtain incredibly more accurate results. When we remove the first error term $E_{1, n}$, by $n=10$ the approximation is about the same as at $n=60$ without the small alteration, and significantly more accurate at the boundary. Subtracting out the next term in the error expansion one sees an equally impressive improvement of accuracy, where with $n=30$ we have at least eight digits of accuracy throughout the interval, and nine digits within the interior.

\section{Extrapolation}

The major failing in using this asymptotic expansion for convergence acceleration is that it requires the odd derivatives of $f$ at the endpoints. Often a function is only given as data, and so derivatives are not directly available. Furthermore, computation of derivatives is a nontrivial task. An alternative method for convergence acceleration is the Gegenbauer reconstruction method [5], but care must be taken to ensure convergence [3].

The knowledge of an asymptotic expansion presents an intriguing possibilty: use extrapolation to increase the convergence rate. The first method one might think of, which was suggested to the author by Daan Huybrechs, would be to evaluate the series at multiple values of $n$ and use a Richardson-like extrapolation method. From Theorem 3.2 we know that

$$
f_{n}(x)=f(x)-A_{1}^{C} \tilde{\Phi}_{1}(x, 1, n)-A_{1}^{S} \tilde{\Phi}_{2}(x, 1, n)+\mathcal{O}\left(n^{-4}\right) .
$$


But this equality holds true for other values of $n$, say, $n_{1}$ and $n_{2}$. Consider the linear combination with unknown constants $r_{1}$ and $r_{2}$ (depending on $n$ and $x$ )

$$
\begin{aligned}
f_{n}(x)+r_{1} f_{n_{1}}(x)+r_{2} f_{n_{2}}(x) & =\left(1+r_{1}+r_{2}\right) f(x) \\
- & A_{1}^{C}\left[\tilde{\Phi}_{1}(x, 1, n)+r_{1} \tilde{\Phi}_{1}\left(x, 1, n_{1}\right)+r_{2} \tilde{\Phi}_{1}\left(x, 1, n_{2}\right)\right] \\
- & A_{1}^{S}\left[\tilde{\Phi}_{2}(x, 1, n)+r_{1} \tilde{\Phi}_{2}\left(x, 1, n_{1}\right)+r_{2} \tilde{\Phi}_{2}\left(x, 1, n_{2}\right)\right] \\
+ & \mathcal{O}\left(n^{-4}\right) .
\end{aligned}
$$

We want to choose $r_{1}$ and $r_{2}$ so that the terms multiplied by the constants $A_{1}^{C}$ and $A_{1}^{S}$ are cancelled. In other words, we determine $r_{1}$ and $r_{2}$ by solving the system

$$
\begin{aligned}
& \tilde{\Phi}_{1}(x, 1, n)+r_{1} \tilde{\Phi}_{1}\left(x, 1, n_{1}\right)+r_{2} \tilde{\Phi}_{1}\left(x, 1, n_{2}\right)=0, \\
& \tilde{\Phi}_{2}(x, 1, n)+r_{1} \tilde{\Phi}_{2}\left(x, 1, n_{1}\right)+r_{2} \tilde{\Phi}_{2}\left(x, 1, n_{2}\right)=0 .
\end{aligned}
$$

Doing so results in $r_{1}$ and $r_{2}$ which depend on $x, n, n_{1}, n_{2}$ and the Lerch transcendent function, but crucially not on the function $f$. We thus obtain the approximation

$$
f_{n}^{R}(x)=\frac{f_{n}(x)+r_{1} f_{n_{1}}(x)+r_{2} f_{n_{2}}(x)}{1+r_{1}+r_{2}} .
$$

This does indeed work pointwise, and surprisingly, we achieve a higher rate of convergence than expected with a specific choice of $n_{1}$ and $n_{2}$ :

Theorem 4.1. Suppose that $n_{1}=n+1$ and $n_{2}=n+2$. For $-1<x<1, a$ sufficiently large $n$ assures that (4.1) has a solution, $1+r_{1}+r_{2} \neq 0$ and that

$$
f_{n}^{R}(x)-f(x)=\mathcal{O}\left(n^{-5}\right) .
$$

Proof. Once we prove that $1+r_{1}+r_{2}$ does not vanish and $r_{1}, r_{2}$ are bounded for large $n$, we know from the construction of $f_{n}^{R}(x)$ that

$$
\begin{aligned}
& f(x)-f_{n}^{R}(x) \\
& =\frac{1}{1+r_{1}+r_{2}}\left\{A_{2}^{C}\left[\tilde{\Phi}_{1}(x, 2, n)+r_{1} \tilde{\Phi}_{1}(x, 2, n+1)+r_{2} \tilde{\Phi}_{1}(x, 2, n+2)\right]\right. \\
& +A_{2}^{S}\left[\tilde{\Phi}_{2}(x, 2, n)+r_{1} \tilde{\Phi}_{2}(x, 2, n+1)+r_{2} \tilde{\Phi}_{2}(x, 2, n+2)\right] \\
& \left.+\mathcal{O}\left(n^{-6}\right)\right\} \text {. }
\end{aligned}
$$

From Cramer's rule, we know that

$$
r_{1}=\frac{\operatorname{det}\left(\begin{array}{ll}
-\tilde{\Phi}_{1}(x, 1, n) & \tilde{\Phi}_{1}\left(x, 1, n_{2}\right) \\
-\tilde{\Phi}_{2}(x, 1, n) & \tilde{\Phi}_{2}\left(x, 1, n_{2}\right)
\end{array}\right)}{\operatorname{det}\left(\begin{array}{ll}
\tilde{\Phi}_{1}\left(x, 1, n_{1}\right) & \tilde{\Phi}_{1}\left(x, 1, n_{2}\right) \\
\tilde{\Phi}_{2}\left(x, 1, n_{1}\right) & \tilde{\Phi}_{2}\left(x, 1, n_{2}\right)
\end{array}\right)} .
$$


From Lemma 3.1 we find that

$$
\begin{aligned}
(-1)^{n} \tilde{\Phi}_{1}(x, 1, n) & =\frac{e^{i \pi(n+1) x}}{\left(1+e^{i \pi x}\right) n^{2}}+\frac{e^{-i \pi(n+1) x}}{\left(1+e^{-i \pi x}\right) n^{2}}+\mathcal{O}\left(n^{-3}\right) \\
& =\frac{1}{n^{2}} \cos \left(n+\frac{1}{2}\right) \pi x \sec \frac{\pi x}{2}+\mathcal{O}\left(n^{-3}\right), \\
(-1)^{n} \tilde{\Phi}_{2}(x, 1, n) & =\frac{e^{i \pi\left(n+\frac{1}{2}\right) x}}{\left(1+e^{i \pi x}\right) n^{2}}-\frac{e^{-i \pi\left(n+\frac{1}{2}\right) x}}{\left(1+e^{-i \pi x}\right) n^{2}}+\mathcal{O}\left(n^{-3}\right) \\
& =\frac{i}{n^{2}} \sin n \pi x \sec \frac{\pi x}{2}+\mathcal{O}\left(n^{-3}\right) .
\end{aligned}
$$

Thus, assuming $n$ is large enough that the denominator does not vanish,

$$
\begin{aligned}
r_{1} & =-\frac{(-1)^{2 n+2} \frac{\sec ^{2} \frac{\pi x}{2}}{n^{4}} \operatorname{det}\left(\begin{array}{cc}
\cos \left(n+\frac{1}{2}\right) \pi x & \cos \left(n+\frac{5}{2}\right) \pi x \\
\sin n \pi x & \sin (n+2) \pi x
\end{array}\right)+\mathcal{O}\left(n^{-5}\right)}{(-1)^{2 n+3} \frac{\sec ^{2} \frac{\pi x}{2}}{n^{4}}\left(\begin{array}{cc}
\cos \left(n+\frac{3}{2}\right) \pi x & \cos \left(n+\frac{5}{2}\right) \pi x \\
\sin (n+1) \pi x & \sin (n+2) \pi x
\end{array}\right)+\mathcal{O}\left(n^{-5}\right)} \\
& =2 \cos \pi x+\mathcal{O}\left(n^{-1}\right) .
\end{aligned}
$$

Similar logic shows that $r_{2}=1+\mathcal{O}\left(n^{-1}\right)$. Thus $1+r_{1}+r_{2}=2+2 \cos \pi x+\mathcal{O}\left(n^{-1}\right)$ is nonzero for large $n$ (since $x \neq \pm 1$ ).

Again from Lemma 3.1 we know that

$$
\begin{aligned}
(-1)^{n} \tilde{\Phi}_{1}(x, 2, n) & =\frac{e^{i \pi(n+1) x}}{\left(1+e^{i \pi x}\right) n^{4}}+\frac{e^{-i \pi(n+1) x}}{\left(1+e^{-i \pi x}\right) n^{4}}+\mathcal{O}\left(n^{-5}\right) \\
& =\frac{1}{n^{4}} \cos \left(n+\frac{1}{2}\right) \pi x \sec \frac{\pi x}{2}+\mathcal{O}\left(n^{-5}\right) .
\end{aligned}
$$

A trivial trigonometric rearrangement informs us that

$$
\cos \left(n+\frac{1}{2}\right) \pi x-2 \cos \pi x \cos \left(n+\frac{3}{2}\right) \pi x+\cos \left(n+\frac{5}{2}\right) \pi x=0
$$

Thus

$$
\begin{aligned}
& \tilde{\Phi}_{1}(x, 2, n)+r_{1} \tilde{\Phi}_{1}(x, 2, n+1)+r_{2} \tilde{\Phi}_{1}(x, 2, n+2) \\
& =\tilde{\Phi}_{1}(x, 2, n)+2 \cos \pi x \tilde{\Phi}_{1}(x, 2, n+1)+\tilde{\Phi}_{1}(x, 2, n+2)+\mathcal{O}\left(n^{-5}\right) \\
& =\frac{(-1)^{n}}{n^{4}} \sec \frac{\pi x}{2}\left[\cos \left(n+\frac{1}{2}\right) \pi x-2 \cos \pi x \cos \left(n+\frac{3}{2}\right) \pi x e^{i \pi}+\cos \left(n+\frac{5}{2}\right) \pi x e^{2 i \pi}\right] \\
& \quad \quad+\mathcal{O}\left(n^{-5}\right) \\
& =\mathcal{O}\left(n^{-5}\right) .
\end{aligned}
$$

Similar logic shows that

$$
\tilde{\Phi}_{2}(x, 2, n)+r_{1} \tilde{\Phi}_{2}(x, 2, n+1)+r_{2} \tilde{\Phi}_{2}(x, 2, n+2)=\mathcal{O}\left(n^{-5}\right)
$$

as well, which completes the proof.

The left graph of Figure 5 demonstrates numerically that the error does indeed have the predicted rate of decay. Unfortunately, the proof only holds true for fixed values of $x$ : we showed that the approximation is bounded for large $n$, but what "large" means depends on $x$. This is demonstrated in the right graph, which shows that the approximation is not uniform, and that the error blows up at certain values of $x$ for fixed $n$. It is possible to prove that this extrapolation converges like $\mathcal{O}\left(n^{-3}\right)$ 

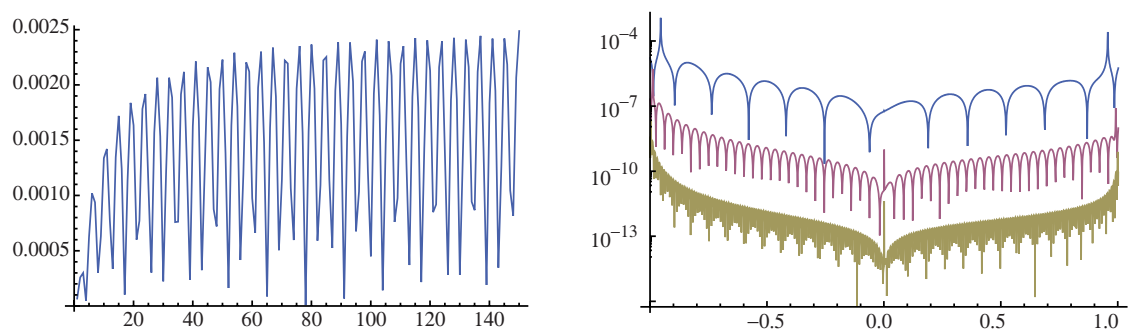

Figure 5. In the left graph, we plot $n^{5}\left|\mathrm{Ai}(0.23)-f_{n}^{R}(0.23)\right|$. In the right graph is shown $\left|\operatorname{Ai}(x)-f_{n}^{R}(x)\right|$ for $n=5,25,100$.

at the endpoints $x= \pm 1$, though we omit the proof since $f_{n}^{R}$ is not accurate within neighbourhoods of these endpoints.

As an alternative, we can treat the coefficients of the asymptotic expansion as unknowns, determined by interpolating the error $f(x)-f_{n}(x)$ by the basis $\left\{\tilde{\Phi}_{1}(x, 1, n), \tilde{\Phi}_{2}(x, 1, n), \tilde{\Phi}_{1}(x, 2, n), \tilde{\Phi}_{2}(x, 2, n), \ldots\right\}$. There is one clear problem, however: this basis is not a Chebyshev set; thus there is no guarantee of interpolation. To circumvent this issue, we employ least squares at a sequence of points $\left\{x_{1}, \ldots, x_{\nu}\right\}$.

It turns out that this method does indeed increase the asymptotic order, but now using only information about $f$, not its derivatives. This is proved in the following theorem, which is phrased in a more general framework. In this case, we consider least squares of the function $g_{n}$ by a general basis $\left\{\psi_{1, n}, \ldots, \psi_{m, n}\right\}$. In other words, we define

$$
\mathcal{P}[g]=\left(\begin{array}{c}
g\left(x_{1}\right) \\
\vdots \\
g\left(x_{\nu}\right)
\end{array}\right), \quad A=\mathcal{P}\left[\psi_{1, n}, \ldots, \psi_{m, n}\right] \quad \text { and } \quad \mathbf{b}=\mathcal{P}\left[g_{n}\right] .
$$

Then, for the vector $\mathbf{d}=\left(d_{1}, \ldots, d_{m}\right)^{\top}$ defined by $\mathbf{d}=A^{+} \mathbf{b}$, where $A^{+}$is the Moore-Penrose pseudoinverse of $A$, which minimizes the norm of $\|A \mathbf{d}-\mathbf{b}\|$, we approximate $g_{n}$ by

$$
v_{n}=\sum d_{k} \psi_{k, n}
$$

Theorem 4.2. Suppose that

$$
g_{n}(x)=\sum_{k=1}^{m} a_{k} \psi_{k, n}(x)+\mathcal{O}\left(\alpha_{n, m}\right), \quad \text { where } \alpha_{n, m}=o\left(\alpha_{n, m-1}\right), n \rightarrow \infty .
$$

Let $r$ be the rank of the Vandermonde-type matrix A. Then

$$
g_{n}(x)=v_{n}(x)+\mathcal{O}\left(\alpha_{n, r}\right) .
$$

Proof. First suppose that $\left\|A^{+}\right\|$is bounded as $n \rightarrow \infty$. From the singular value decomposition we know that $A^{+} A=I_{r}$, where $I_{r}$ is the identity matrix with only the first $r$ nonzero elements. Thence

$$
\begin{aligned}
\mathbf{d} & =A^{+} \mathbf{b}=\sum_{k=1}^{m} a_{k} A^{+} \mathcal{P}\left[\psi_{k, n}\right]+A^{+} \mathcal{P}\left[\mathcal{O}\left(\alpha_{n, m}\right)\right] \\
& =A^{+} A \mathbf{c}+\mathcal{O}\left(\alpha_{n, m}\right)=I_{r} \mathbf{c}+\mathcal{O}\left(\alpha_{n, m}\right)
\end{aligned}
$$



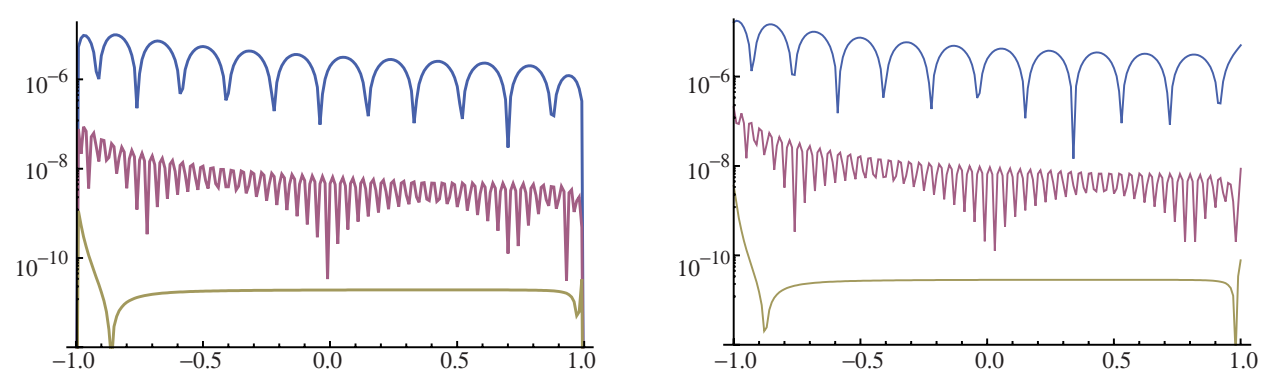

Figure 6 . The error in approximating $\operatorname{Ai}(x)$ by least squares (left) and least squares with Richardson (right), for $n=5,25,100$.

Thus

$$
v(x)=\sum_{k=1}^{m} d_{k} \psi_{k, n}(x)=\sum_{k=1}^{r} a_{k} \psi_{k, n}(x)+\mathcal{O}\left(\alpha_{n, m}\right)=g_{n}(x)+\mathcal{O}\left(\alpha_{n, r}\right) .
$$

If $\left\|A^{+}\right\|$is not bounded for increasing $n$, replacing each $\psi_{k, n}$ by $\left\|A^{+}\right\| \psi_{k, n}$ and each $a_{k}$ by $a_{k} /\left\|A^{+}\right\|$rectifies the problem.

Corollary 4.3. Let

$$
v_{n}(x)=\sum_{k=1}^{m}\left[d_{1, k} \tilde{\Phi}_{1}(x, k, n)+d_{2, k} \tilde{\Phi}_{2}(x, k, n)\right] .
$$

Determine the coefficients via a least squares fit of $f-f_{n}$ at a sequence of points $\left\{x_{1}, \ldots, x_{\nu}\right\}$, where all the points are within the interior interval. Then

$$
f(x)=f_{n}(x)+v_{n}(x)+\mathcal{O}\left(n^{-r-2}\right),
$$

where $r$ is the rank of the matrix

$$
\mathcal{P}\left[\tilde{\Phi}_{1}(x, 1, n), \tilde{\Phi}_{2}(x, 1, n), \ldots, \tilde{\Phi}_{1}(x, m, n), \tilde{\Phi}_{2}(x, m, n)\right] .
$$

Though we have successfully rid ourselves of the dependence of knowing the derivatives of $f$ in order to accelerate convergence, we still need to know the values of $f$ at the interpolation points. This compares to the Richardson-like extrapolation method of Theorem 4.1 which achieved convergence acceleration pointwise by only using the series' coefficients, but not in a uniform manner. The benefits of only using the coefficients themselves are manifold: one can perform manipulations on the series without worrying about the extra asymptotic terms and for compression no additional data needs to be stored. But the interpolation method only needs $f$, or rather, something asymptotic to $f$, at a small number of points, and we know that $f_{n}^{R}$ approximates $f$ well at fixed points; thus we can combine the two methods! Hence, instead of interpolating $f-f_{n}$, we can interpolate $f_{n}^{R}-f_{n}$. Figure 6 compares the least squares method to least squares plus Richardson extrapolation. Both methods use evenly spaced points and achieve an error of order $\mathcal{O}\left(n^{-4}\right)$ at interior points. 


\section{Computation of SeRies COefFicients}

Up until now we have avoided computing the coefficients $c_{k}$ and $s_{k}$ of the modified Fourier series; we merely assumed that they were given. When the function $f$ is well-behaved, i.e., nonoscillatory, the first few coefficients are well-behaved integrals, meaning standard quadrature methods such as Gauss-Legendre quadrature will approximate these integrals efficiently. As $k$ becomes large, the coefficients become highly oscillatory integrals, and the standard methods are no longer efficient. With a bit of finesse, we can successfully compute these integrals in an efficient manner. In fact, using the right quadrature methods, the accuracy improves as the frequency of oscillations increases.

The most well-known method for approximating highly oscillatory integrals is to use the asymptotic expansion, which we have already derived in (2.2). Using a partial sum of the expansion, we obtain an approximation which is more accurate when the frequency of oscillations is large. In fact, when $f$ is sufficiently differentiable, we can obtain an approximation of arbitrarily high asymptotic order. Unfortunately, the asymptotic expansion does not in general converge for fixed values of $k$, meaning that the accuracy of this approximation is limited.

To combat this problem we will use Filon-type methods, which were developed in 9] based on the Filon method found in [4. Using such a method for computing the coefficients of a modified Fourier series was first investigated in $[8]$. The idea behind a Filon-type method is to approximate the function $f$ by a polynomial $v=\sum b_{j} x^{j}$, for which the moments

$$
\int_{-1}^{1} x^{j} \cos \pi k x \mathrm{~d} x \quad \text { and } \quad \int_{-1}^{1} x^{j} \sin \pi\left(k-\frac{1}{2}\right) x \mathrm{~d} x
$$

are known explicitly, in terms of the incomplete Gamma function. This is accomplished using Hermite interpolation, where the asymptotic order of the approximation is determined by the multiplicities at the endpoints, as originally noted in [9]. But in our case the asymptotic expansion only depends on odd derivatives; hence we only require interpolation of the odd derivatives at the endpoints to achieve higher asymptotic order approximations.

Thus assume we are given a sequence of nodes $\left\{x_{1}, \ldots, x_{\nu}\right\}$ and multiplicities $\left\{m_{1}, \ldots, m_{\nu}\right\}$. We then find a polynomial $v(x)=\sum b_{j} x^{j}$ which satisfies

$$
v^{\prime}\left(x_{j}\right)=f^{\prime}\left(x_{j}\right), \ldots, v^{\left(2 m_{j}-1\right)}\left(x_{j}\right)=f^{\left(2 m_{j}-1\right)}\left(x_{j}\right), \quad j=1, \ldots, \nu .
$$

It turns out that this decays at the same order as the partial sums of the asymptotic expansion:

Theorem 5.1 ([8]). Suppose that $f \in C^{2 s+1}[-1,1]$ and $f^{(2 s+1)}$ has bounded variation. Then

for $q=\min \left\{m_{1}, m_{\nu}, s\right\}$ and

$$
c_{k}-c_{k}^{F}, s_{k}-s_{k}^{F}=\mathcal{O}\left(k^{-2 q-2}\right),
$$

$$
c_{k}^{F}=\int_{-1}^{1} v(x) \cos \pi k x \mathrm{~d} x \quad \text { and } \quad s_{k}^{F}=\int_{-1}^{1} v(x) \sin \pi\left(k-\frac{1}{2}\right) x \mathrm{~d} x .
$$

Proof. We consider only the terms $c_{k}$, since the proof for the terms $s_{k}$ is virtually the same. The first $2 q$ terms in the asymptotic expansion of

$$
c_{k}-c_{k}^{F}=\int_{-1}^{1}(f(x)-p(x)) \cos \pi k x \mathrm{~d} x
$$


are zero; hence

$$
c_{k}-c_{k}^{F}=\Re\left\{(-i \pi k)^{-2 q-1} \int_{-1}^{1}\left[f^{(2 q+1)}(x)-v^{(2 q+1)}(x)\right] e^{i \pi k x} \mathrm{~d} x\right\} .
$$

The theorem follows since both $f^{(2 q+1)}$ and $v^{(2 q+1)}$ have bounded variation.

This theorem lets us obtain an approximation with the same asymptotic order as the asymptotic expansion, but significantly more accurate, as we can add internal nodes to increase the accuracy. In general, (5.1) is insufficient to uniquely specify a polynomial; thus we use the following construction:

1. Determine the polynomial $w=\sum b_{j} x^{j}$ by solving the system

$$
w\left(x_{j}\right)=f^{\prime}\left(x_{j}\right), \ldots, w^{\left(2 m_{j}\right)}\left(x_{j}\right)=f^{\left(2 m_{j}-1\right)}\left(x_{j}\right), \quad j=1, \ldots, \nu .
$$

2. Define $v=f(0)+\int_{0}^{x} w(t) \mathrm{d} t$.

This leaves us with the slight problem of how to decide when to use oscillatory quadrature methods and when to use nonoscillatory quadrature methods. We avoid investigating this problem as it is too tangential to the subject of this paper. Instead, in our example, we compute the coefficients $c_{0}, \ldots, c_{5}$ and $s_{1}, \ldots, s_{5}$ to machine precision using Gauss-Legendre quadrature, and compute the other coefficients with three different Filon-type methods. A more sophisticated quadrature method exists, namely exotic quadrature [7], which reuses the information already obtained for a Filon-type method to approximate the nonoscillatory coefficients.

We denote the partial sum of the modified Fourier series using the coefficients computed using a Filon-type method as $f_{n}^{F}$, where the choices for nodes and multiplicities are determined by the context. We could use different nodes and multiplicities for different values of $k$, as less information is needed for large $k$ in order to obtain accurate approximations. We however use the same Filon-type method for all $k$ for simplicity. When we replace the exact value of the coefficients with an approximation, we incur an error that cannot be rectified; hence we no longer have convergence. Instead, we converge to the constant

$$
\begin{aligned}
f(x) & -\lim _{n \rightarrow \infty} f_{n}^{F}(x) \\
& =\frac{c_{0}-c_{0}^{F}}{2}+\sum_{k=1}^{\infty}\left[\left(c_{k}-c_{k}^{F}\right) \cos \pi k x+\left(s_{k}-s_{k}^{F}\right) \sin \pi\left(k-\frac{1}{2}\right) x\right] .
\end{aligned}
$$

This sum is finite since $c_{k}^{F}$ and $s_{k}^{F}$ are at least $\mathcal{O}\left(k^{-2}\right)$. As the Filon-type method used becomes more accurate, this constant will decrease.

Returning to the Airy example of Figure 1 with $x=-1 / \sqrt{2}$, we now show the results for three Filon-type methods: endpoints for nodes and multiplicities both one (i.e., only the first derivative is used), nodes $\{-1,-\sqrt{3} / 2,0, \sqrt{3} / 2,1\}$ and multiplicities all one and endpoints for nodes and multiplicities both two (i.e., the first and the third derivative are used). The choice of nodes in the second Filon-type method is simply the Chebyshev-Lobatto points [14]. Note that with or without the correcting term $E_{1, n}(x)$, we converge to the same value (5.2). With this correcting term, however, we converge to this value significantly faster. The last Filon-type method is of a higher order, so the terms in (5.2) go to zero at a quicker rate, and as a result the error is significantly smaller. In this case (5.2) is of the order of $10^{-7}$. 

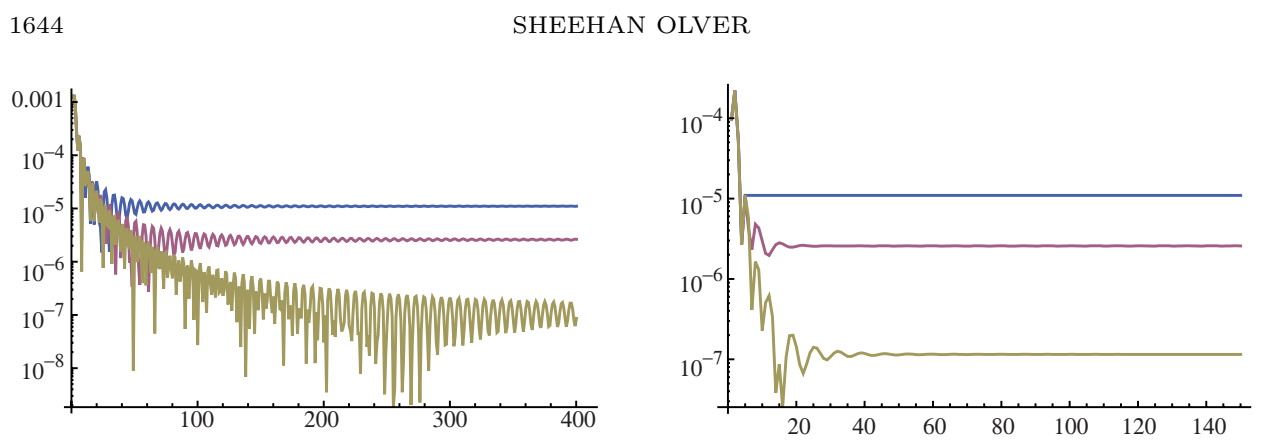

Figure 7. For $f(x)=\operatorname{Ai}(x)$, the error $\left|f(-1 / \sqrt{2})-f_{n}^{F}(-1 / \sqrt{2})\right|$ (left graph) with endpoints for nodes and multiplicities both one (top), nodes $\{-1,-\sqrt{3} / 2,0, \sqrt{3} / 2,1\}$ and multiplicities all one (middle) and endpoints for nodes and multiplicities both two (bottom), compared to the error $\left|f(-1 / \sqrt{2})-f_{n}^{F}(-1 / \sqrt{2})-E_{1, n}(-1 / \sqrt{2})\right|$ with the same choices of Filon-type methods (right graph).

Remark. There are other methods that can be used to compute oscillatory integrals, such as Levin-type methods [13, which are based on the Levin collocation method [11], as well as numerical steepest descent [6].

\section{Future WORK}

The most immediate open question is whether we can determine a more general class of functions which converge at the rate of $\mathcal{O}\left(n^{-2}\right)$. We know it cannot be increased to $L_{2}[-1,1]$, since, as an example, the function $\operatorname{sgn} x$ does not have coefficients which decay like $\mathcal{O}\left(k^{-2}\right)$ :

$$
\begin{aligned}
s_{k} & =\left\langle\operatorname{sgn}, \sin \pi\left(k-\frac{1}{2}\right) x\right\rangle=\int_{0}^{1} \sin \pi\left(k-\frac{1}{2}\right) x \mathrm{~d} x-\int_{-1}^{0} \sin \pi\left(k-\frac{1}{2}\right) x \mathrm{~d} x \\
& =-\frac{4}{\pi(1-2 k)} .
\end{aligned}
$$

Substituting this into the proof of Theorem 3.2 results in an infinite sum that reduces to the Lerch transcendent function $\Phi\left(-e^{-i \pi x}, 1, n+\frac{1}{2}\right)$. Since $s=1$, it is known that this decays no faster than $\mathcal{O}\left(n^{-1}\right)$. But it might be possible to impose conditions similar to bounded variation on a $C^{0}$ or $C^{1}$ function in order to guarantee the faster rate of convergence.

Consider again the eigenvalue problem from which we obtained the basis in our orthogonal series:

$$
u^{\prime \prime}+\alpha^{2} u=0, \quad 0=u^{\prime}(1)=u^{\prime}(-1) .
$$

In [7, a generalization of this eigenvalue problem was explored:

$$
u^{(2 q)}+(-1)^{q+1} \alpha^{2 q} u=0, \quad 0=u^{(i)}(-1)=u^{(i)}(1), \quad i=q, q+1, \ldots, 2 q-1 .
$$

The solutions of this problem, namely the polyharmonic eigenfunctions [10], lead to a new orthogonal series which is dense in $L_{2}[-1,1]$, and whose coefficients decay like $\mathcal{O}\left(n^{-q-1}\right)$ for sufficiently differentiable functions. When $q$ is one, we again obtain the modified Fourier series we have analysed. Choosing $q$ larger than one, on the other hand, gives us a series whose coefficients decay at an even faster rate. 
It might be possible to prove the convergence rate of this series in the same manner as we did in Theorem 3.2. Unfortunately, pointwise convergence of this series has not been proved, which would be necessary in order to rewrite the error as an infinite sum. Some initial results have been found for generalizing polyharmonic eigenfunctions for the approximation of multivariate functions. It may also be possible to generalize the results of this paper to prove the convergence rate in multivariate domains.

\section{ACKNOWLEDGMENTS}

The author would like to thank his Ph.D. supervisor Arieh Iserles (University of Cambridge) and Syvert Nørsett (NTNU), for introducing him to this new area of research and numerous helpful suggestions. He would also like to thank Ben Adcock (University of Cambridge), Daan Huybrechs (K.U. Leuven), Tom Körner (University of Cambridge) and Alexei Shadrin (University of Cambridge).

\section{REFERENCES}

[1] Abramowitz, M., Stegun, I., Handbook of Mathematical Functions with Formulas, Graphs, and Mathematical Tables, National Bureau of Standards Appl. Math. Series, \#55, U.S. Govt. Printing Office, Washington, D.C., 1964. MR0167642 (29:4914)

[2] Aksenov, S., Savageau, M.A., Jentschura, U.D., Becher, J., Soff, G., Mohr, P.J., Application of the combined nonlinear-condensation transformation to problems in statistical analysis and theoretical physics, Comp. Phys. Comm., 150 (2003) 1-20.

[3] Boyd, J.P., Trouble with Gegenbauer reconstruction for defeating Gibbs' phenomenon: Runge phenomenon in the diagonal limit of Gegenbauer polynomial approximations, J. Comput. Physics, 204 (2005) 253-264. MR.2121910 (2005m:65005)

[4] Filon, L.N.G., On a quadrature formula for trigonometric integrals, Proc. Roy. Soc. Edinburgh 49 (1928) 38-47.

[5] Gottlieb, D., Shu, C.-W., On the Gibbs phenomenon and its resolution, SIAM Review 39 (1997) 644-668. MR.1491051 (98m:42002)

[6] Huybrechs, D., Vandewalle, S., On the evaluation of highly oscillatory integrals by analytic continuation, SIAM J. Numer. Anal. 44 (2006) 1026-1048. MR 2231854 (2007d:41033)

[7] Iserles, A., Nørsett, S.P., From high oscillation to rapid approximation II: Expansions in polyharmonic eigenfunctions, DAMTP Tech. Rep. NA2006/07.

[8] Iserles, A., Nørsett, S.P., From high oscillation to rapid approximation I: Modified Fourier expansions, IMA J. Numer. Anal. 28 (2008), 862-887. MR2457350

[9] Iserles, A., Nørsett, S.P., Efficient quadrature of highly oscillatory integrals using derivatives, Proceedings Royal Soc. A. 461 (2005) 1383-1399. MR2147752(2006b:65030)

[10] Krein, M. G., On a special class of differential operators, Doklady AN USSR 2 (1935), 345349.

[11] Levin, D., Procedures for computing one and two-dimensional integrals of functions with rapid irregular oscillations, Math. Comp. 38 (1982), no. 158, 531-538. MR645668|(83a:65023)

[12] Olver, F.W.J., Asymptotics and Special Functions, Academic Press, New York, 1974. MR0435697(55:8655)

[13] Olver, S.; Moment-free numerical integration of highly oscillatory functions, IMA J. Numer. Anal. 26 (2006) 213-227. MR2218631 (2006k:65064)

[14] Powell, M.J.D., Approximation Theory and Methods, Cambridge University Press, Cambridge, 1981. MR604014 (82f:41001)

[15] Srivastava, H.M., Choi, J., Series associated with the zeta and related functions, Kluwer Academic Publishers, Dordrecht, The Netherlands, 2001. MR1849375 (2003a:11107)

Oxford University Computing Laboratory, Wolfson Building, Parks Road, Oxford, UNITED Kingdom

E-mail address: sheehan.olver@sjc.ox.ac.uk 